\title{
Virtualization Approach: Theory and Application
}

\author{
Hamdani $^{1}$, Andysah Putera Utama Siahaan ${ }^{2}$ \\ ${ }^{I}$ Faculty of Engineering, Universitas Pembangunan Panca Budi, Medan, Indonesia \\ ${ }^{2}$ Faculty of Computer Science,Universitas Pembangunan Panca Budi, Medan, Indonesia
}

\begin{abstract}
Virtualization is a new method regarding using computing resources efficiently, by maximizing energy efficiency, extend the life of the hardware and also recycles. Virtualization technology is a system of work done by the software can merge some real physical systems into a single virtual form commonly known as virtualization without prejudice advantages over the single system. Of course, this system can reduce the amount of hardware, electrical energy consumption and time used thereby increasing the level of efficiency and effectiveness. Also, virtualization certainly reduces heat energy arising from the number of installed hardware, thereby reducing the increase in temperature geothermal (Global Warming). Some virtualization includes Server Virtualization, Network Virtualization, Memory Virtualization, Grid Computing, Application Virtualization, Storage Virtualization, Virtualization and Thin Client Platform.
\end{abstract}

Keywords: Virtualization, Virtual Machine

\section{Introduction}

Virtualization is a method that emulates a real system into a virtual system that physically separated dependence. In other words, something that has been virtualized can no longer be seen with the eye or held, but has the same function as the original system. The purpose of virtualization is to make savings from all aspects. Virtualization is a concept that was first developed in the 1960s to manage mainframe hardware. And also in 1960-based computer x86 architecture are faced with the same problem, namely rigidity and underutilization.Virtualization is always used first of all just to test the invention or new system over an existing system. It aims to get results or to test whether the new system has still to be compatible or needed further improvement. Then some subsequent period virtualization can stand alone in a parent (host) system. This understanding tries to describe the virtualization technique. It describes the basic rule of the virtuality.

\subsection{Virtual Machine}

\section{Theories}

Virtual machine (VM) is a device that typically is a software or operating system, which can not be seen physically but can be run in an environment other. In this case, the VM is referred to as a "guest" because the device only rides, while the environment is running so-called "host." The basic concept of the virtual machine is that it emulates the hardware of a computer consisting of a CPU, memory, HDD into multiple execution environments, so that seems to have the physical device on each virtualization. VM to appear for their trial to run multiple operating systems on a single computer.

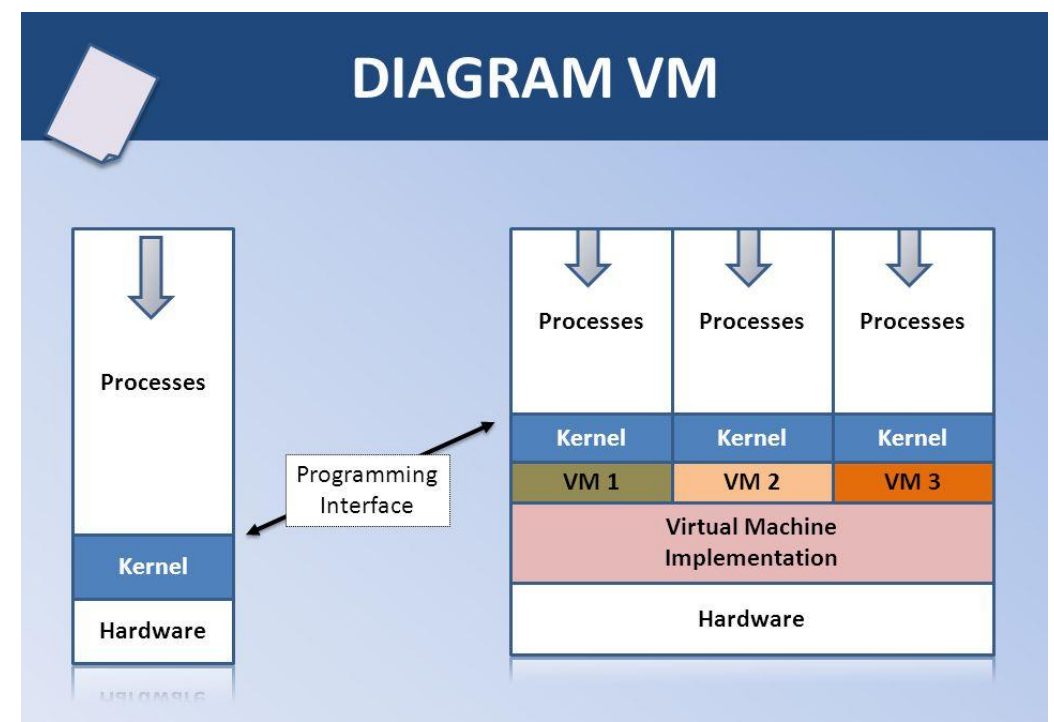

Fig. 1 Virtual Machine Architecture 
Figure 1 shows the virtual machine architecture. The server can covers several virtual machines simultaneously. Virtual machine technology has many functions such as hardware consolidation, facilitate the rescue system, and run the software earlier. One of the most important applications of virtual machine technology is cross-platform integration. Some other important applications are:

1. Server Consolidation. If several servers run applications that only takes up very little resources, VM can be used to incorporate the applications that run on only one server, even if the application requires that the operating system is different.

2. Automation and consolidation. Each VM can act as different environments; this allows developers so no need to provide environment physically.

3. Easy System Recovery. Virtualization can be used for system recovery that requires portability and flexibility across platforms.

4. The demonstration software. With VM technology, clean operating system, and its configuration can be provided quickly.

\section{A. Virtualization Types}

Virtual Machine provides convenience regarding the distribution of hardware resources that exist in each guest system, each running a guest operating system itself. The software provides the virtualization is called a virtual machine monitor or hypervisor. A hypervisor can run on hardware or an operating system.

The main advantages and VM system are:

1. Various operating system environment can be run on the same computer, in strong isolation between environments

2. VM can provide the instruction set architecture (ISA) that is different to that of the hardware.

The operating system on the guest does not have the same operating system. The use of the VM to support a variety of different operating systems became so popular in embedded systems, where the real-time operating system is used in conjunction with a high-level operating system such as Linux or Windows. Another function is to sandbox that isolates changes in the codes that suspicion arises from the OS that can not be trusted because it is still in the development stage. VM has other benefits in the development of operating systems such as access better debugging and reboots faster than the original machine.

\subsection{Virtualization Process}

A virtual machine process sometimes called an application virtual machine, runs as a normal application inside an operating system and supports the process. VM process was created at the time the process was initiated and is destroyed when the process exits. The aim is to provide a programming environment that is platform-independent which abstracts the details of the software or operating system and allow a program executed in the same way on any platform. The process VM provides a high-level abstraction process is implemented clicking VM-use interpreters. VM-type became popular with the Java programming language, which is implemented by the Java Virtual Machine. Another example is the .NET Framework, which runs on a VM called the Common Language Runtime. A special case of the VM process is a system that abstracts the communication mechanism of cluster computers. VMnya is not a single process, but rather one process per physical machine in the cluster. VM is designed to simplify the work program an application in parallel with letting the programmer focus on algorithms rather than the communication mechanisms provided by the interconnect and operating systems. The fact that communication was not hidden and the cluster is not cultivated represented as a single machine.

\subsection{Virtualization Sample}

In GNU / Linux, one is the famous virtual machine is Vmware. VMware allows multiple operating systems to run on one machine PC simultaneously. It can be done without having to reconfigure the storage media and reboot. On the Virtual Machine supplied will be executed by the operating system as desired. In this way, the user can boot up an operating system Linux as the host operating system and then run other operating systems, such as Microsoft Windows or Solaris. The operating system that runs on the host operating system is known as the guest operating system. 


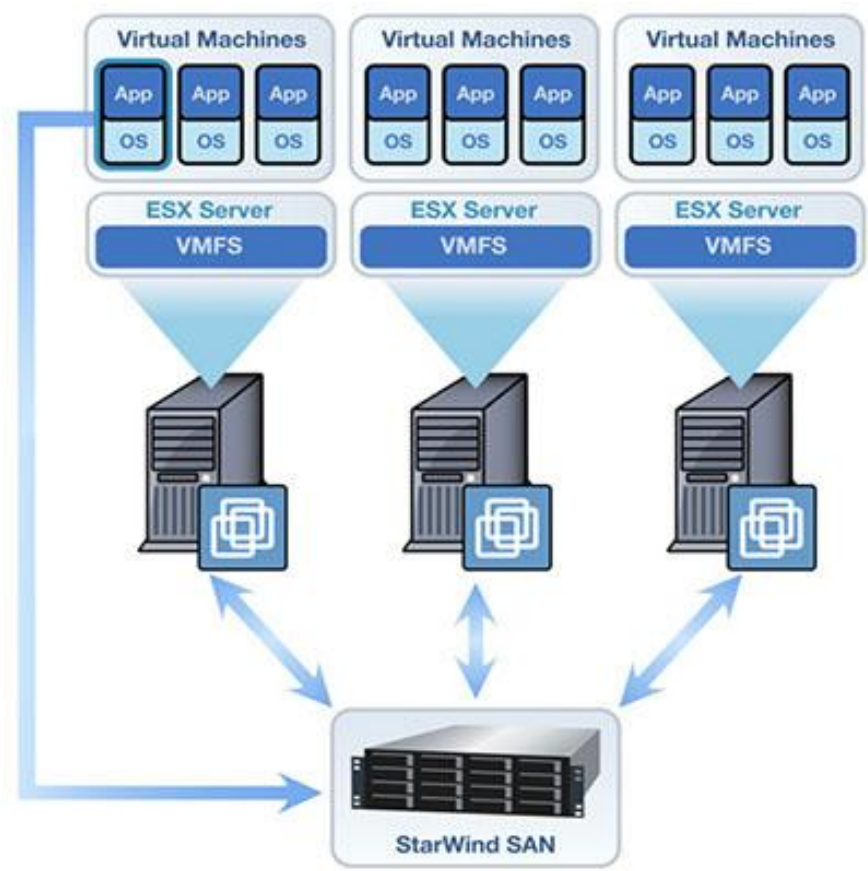

Fig. 2VMware Virtual Machine Scheme

VMware logically regarded as software is often used for the experimental new operating system, games, applications, to install two operating systems and run (cross platform) on the same drive without having to dualboot. Technically we just press Alt + Tab to switch operating systems. But VMware is not the emulator as well as PS2 emulator because it does not emulate a CPU and hardware in a Virtual Machine (VM), but only allow other operating systems to run in parallel with the operating system has been running. Each Virtual Machine (VM) can have its IP address that can be tested connections of the connected network (ping). The sample of this can be seen in Figure 2. It describes the scheme of the virtualization usage.

\section{Implementation}

Before the virtualization runs, the operating system must first be executed first. If the operating system on a computer having problems, eating the worst thing to do is reinstall. While in the world of virtualization everything changed 180 degrees.

\subsection{Full Virtualization}

Full Virtualization is a way of doing virtualization for the various virtual machine environment, where the model of full virtualization provides a complete modeling of hardware. The complete simulation allows any software that can be executed directly on the host operating system can also be executed on the guest operating systems in, including all operating systems.One illustration of full virtualization is a control program of the operating system CP / CMS from IBM. Each user of the CP / CMS given a computer system (which is a virtual machine) that stands alone. The virtual machine has all the capabilities of the underlying hardware, and to its users, the virtual machine can not be distinguished by a system of its own. Simulations carried out thoroughly and based on the principle of operation of hardware that includes instruction sets, main memory, interrupts, exceptions, and device access. A result is a machine that can be shared among many users.Full virtualization is only possible with the right combination of hardware and the right software. For example, it is not possible for most systems IBM System / 360. X86 systems also were once thought not to be able to run full virtualization, but by using the binary technique translation, VMware can run full virtualization.

The main challenge in full virtualization is the simulation of the operation that requires special privileges such as instruction M / K. The effect of any operations performed in the VM must be kept inside the VM - virtual operation is not allowed to change the status in another VM, the control program, or hardware. Instructions whose influence is regulated by the control program can be directly executed by the hardware. While the instructions that could affect up to outside the VM must be wrapped and simulated. Full virtualization has so far proved a success for the division of a computer system to be used for the isolation of many users and users with other users and with the control program to obtain the reliability and security of the system. 


\subsection{Half Virtualization}

Half Virtualization in computer science is a virtualization technique that is used for implementation on a wide variety of virtual machine environment, which in this part of VM virtualization environment only provides partial hardware simulation only. Not all of the hardware features simulated so that not all software can run without modification first.The primary key of the partial address virtualization is virtualization, which means that each virtual machine consists of an address independent. This ability should be supported by the ability to relocate from a hardware address - already exists in most practical implementations of virtualization half.Virtualization is the initial part of their full virtualization. Virtualization part used in the first generation of time-sharing systems CTSS and experimental paging system on the IBM M44 / 44x. This term can also be used to describe the operating system that provides separate address space for different users or processes.Virtualization half is much more easy to implement than full virtualization, often unable to provide a useful and powerful VM and supports critical applications. The drawback is a hardware compatibility issue earlier and portability (not multiple systems). If hardware features are not simulated, then the software that uses the feature will fail to run.

\subsection{Native Virtualization}

Native Virtualization is a technique by which a VM is used to simulate a complete hardware environment so that the operating system can run unmodified on the same CPU type in complete isolation in the VM container. Native virtualization leveraging the capabilities of hardware support available in the latest processors from Intel (Intel VT) and Advanced Micro Devices (AMD-V) to provide performance closer to the original system.Native virtualization, also known as accelerated virtualization or virtualization hybrid is a combination of full virtualization and acceleration techniques I / $\mathrm{O}$ and is often used to greatly improve the performance of full virtualization. Typically, this method starts with the Virtual Machine Monitor is capable of full virtualization ago, based on analysis of performance, running acceleration technique chosen. I / O and network drivers are part of the most common virtualization accelerated in the original.

\section{Virtualization and Probabilities}

In the past, the boundaries between virtualization and portability are fairly clear boundaries. When you hear the word virtualization of computing, always first imagined virtual memory, and virtual machine (like Java) or virtual environment (Operating System running on other operating systems). But not imagined that virtualization can be attributed to its portability. Portability is more complex than just a program that can be moved easily and may be made without too much reconfiguration in another environment. Portability in a Windows environment which I am most familiar is the Portable Application Suite, in the Linux environment is already so familiar it feels us with LiveCD, LiveDVDs, and LiveUSB.Currently, there are so many alternatives to VMware as virtualization. From the world of open source, we know Xen, QEMU, and VirtualBox Colinux. Even Microsoft also issued a Virtual PC. The most exciting development is all becoming increasingly compact. Sekompak possible to enable the Virtual Disks that have been made can be moved easily. Virtualization is now the case is not too complex. Configuration is easy, and there are many alternatives to VMWare. There are some things that make us need to think further about green computing, matter and energy efficiency.

Virtualization on a more serious computing from the desktop is needed mainly for efficiency. Basically, virtualization has always been that the existence of the operating system. Several processes or software is computing nicer to work in their natural environment or even have software that is only available on the specific operating system platform. For example, the famous AutoCAD drafter environment has not had a substitution outside of Windows. Virtualization also creates other efficiencies, where a machine can be used to run multiple operating systems, or operate system can be run at any time when required by the same machine that was running the other operating system.

\section{Virtualization Softwares}

Before attempting virtualization to note is how adequate capabilities (hardware) computer to virtual desired operating system. The host system needs a large of resources since it shares its resources to the guest operating systems. There are some of the virtualization software available, such as:

\section{1 coLinux}

coLinux or Cooperative Linux is a special Virtualization in Windows for multiple Linux distributions. With coLinux, we can run Ubuntu, Debian, ArchLinux, Fedora and others. The interesting thing is, when the image is placed at the Linux USB Portable Notebook, it did not disturb. I say almost no degradation of performance which means until the process is finished booting Linux. We can bring coLinux daemon without having to be installed on a USB Flash image at the same time we need. 


\section{$4.2 Q E M U$}

QEMU emulator can run as meaning it can run operating systems for one type of machine to machine another kind or as a virtualizationapplication with QEMU accelerator executes guest code (other operating systems) directly to the host (the primary operating system / parent). This is another type of savings. QEMU is more advanced than coLinux because it is available on multiple operating systems.Qemu is equipped with QEMU Manager GUI so that we can arrange the images of operating systems and virtualization that we prepare with ease. Allocate resources, disk space and what devices in parent operating system that will be mapped to the virtual operating system (Guest OS). Defining this should be done with the text mode configuration coLinux. Performance, in some ways, coLinux better, but QEMU has more features than coLinux. coLinux present for Windows users who want to learn Linux. Running Qemu as an emulator, not required complicated procedures. To try an OS, we do not even need to allocate space for the virtual hard disk image; we can even run the iso from the operating system. If we've already downloaded the Ubuntu iso, FreeDOS or the latest Fedora, then we can immediately run with QEMU with only direct CD / DVD to boot the iso. Qemu present as a virtualizes app in many operating systems for computing broadly than just try.

\subsection{VirtualBox}

VirtualBox became part of the SUN. VirtualBox foreclosed SUN SUN to add the portfolio, especially in working on Xen virtualization as Novell bundled into SUSE Linux Enterprise and Microsoft acquired from Connectix VirtualPC. SUN declare VirtualBox virtual computing ready for a more serious so that it can be used from home to enterprise scale.VirtualBox is available on many operating systems (host): Linux, Solaris, and OpenBSD. Virtualbox developed rapidly, releases the series out in a relatively fast also with new features. The concept offered at Full Virtualization in VirtualBox is the same as VMWare, QEMU, and Win4Lin where the hardware specifications of the guest hosts will follow the hardware specifications of increasingly sophisticated increasingly sophisticated hardware host a guest anyway. However, there is some hardware virtualized host alias does not follow that the CD / DVD drive, hard drive, RAM, and VGA memory of the benefits you can manage the ability of the virtualized hardware but it can not exceed the ability of the host hardware.Like a guest requires the original computer RAM and hard drive space. The amount of RAM memory RAM will cut the guest host, are trivial RAM must be allocated to the guest host. For example, a guest requires $256 \mathrm{MB}$ of RAM memory and a memory of $1 \mathrm{~Gb}$ host so the host memory: $256 \mathrm{Mb}$ to $1 \mathrm{~Gb}$ reduced. If there is a guest host, more memory to be allocated again. As for the guest, the disk should not beexceeding the capacity of the host storage. Virtual hard disk files can be stored on a flash drive, a regular hard drive, network and so on, but the virtual disk capacity can not exceed the virtual hard drive where it was saved.

\section{Conclusion}

Virtualization is a method to make something off of physical dependence. Example; a virtual machine is a computer, which was only in the form of a file on the hard disk. With virtualization, then a computer (physical) can run many virtual computers while at the same time. The types of VM is the VM system in which a VM can run its operating system; then the VM is where the VM just to run a single process. Then VM is also divided by the level of virtualization, namely full virtualization which simulates all the features of the hardware that allow software running on a VM without modification. Then virtualization half, where not all of the hardware features simulated. The latter is a native virtualization, which is a full virtualization combined with the help of hardware that supports virtualization.

[1]. P. G and Vijayrajan, "Analysis of Performance in the Virtual Machines Environment," International Journal of Advanced Science and Technology, vol. 32, no. 7, pp. 53-64, 2011.

[2]. Ali dan N. Meghanathan, "Virtual Machines And Networks, Installation, Performance, Study, Advantages and Virtualization Options," International Journal of Network Security \& Its Applications, vol. 3, no. 1, pp. 1-15, 2011.

[3]. S. M., H. G. M., N. A. dan U. J., "Performance Analysis of Kernal-Based Virtual Machine," International Journal of Computer Science \& Information Technology, vol. 5, no. 1, pp. 137-144, 2013.

[4]. X. Zhao, K. Borders dan A. Prakash, Virtual Machine Security Systems, vol. 57, USA: Department of EECS, University of Michigan, 2013, pp. 339-365.

[5]. P. H. Gum, "System/370 extended architecture: Facilities for virtual machines," IBM Journal of Research and Development, vol. 27, no. 6, p. 530-544, 1983 .

[6]. P. F. Silvia, R. Karthiha, R. Aarthy dan C. S. G. Das, "Virtual machine vs Real Machine: Security Systems," International Journal of Engineering and Technology, vol. 1, no. 1, pp. 9-13, 2009.

[7]. A. P. U. Siahaan, "Comparison Analysis of CPU Scheduling : FCFS, SJF and Round Robin," International Journal of Engineering Development and Research, vol. 4, no. 3, pp. 124-132, 2016.

[8]. R. A. Meyer dan L. H. Seawright, “A Virtual Machine Time Sharing System,” IBM System Journal, vol. 9, no. 3, pp. 199-218, 1970. 